\title{
Lichen amyloidosis
}

\section{Barry Ladizinski MD, Kachiu Cecilia Lee MD MPH}

Competing interests: None declared.

This article has been peer reviewed.

Affiliations: Johns Hopkins Bloomberg School of

Public Health (Ladizinski), Baltimore, Md.;

Department of Dermatology (Lee), Brown University,

Providence, RI

Correspondence to:

Barry Ladizinski,

barryladizinski@gmail.com

CMAJ 2014. DOI:10.1503 /cmaj.130698
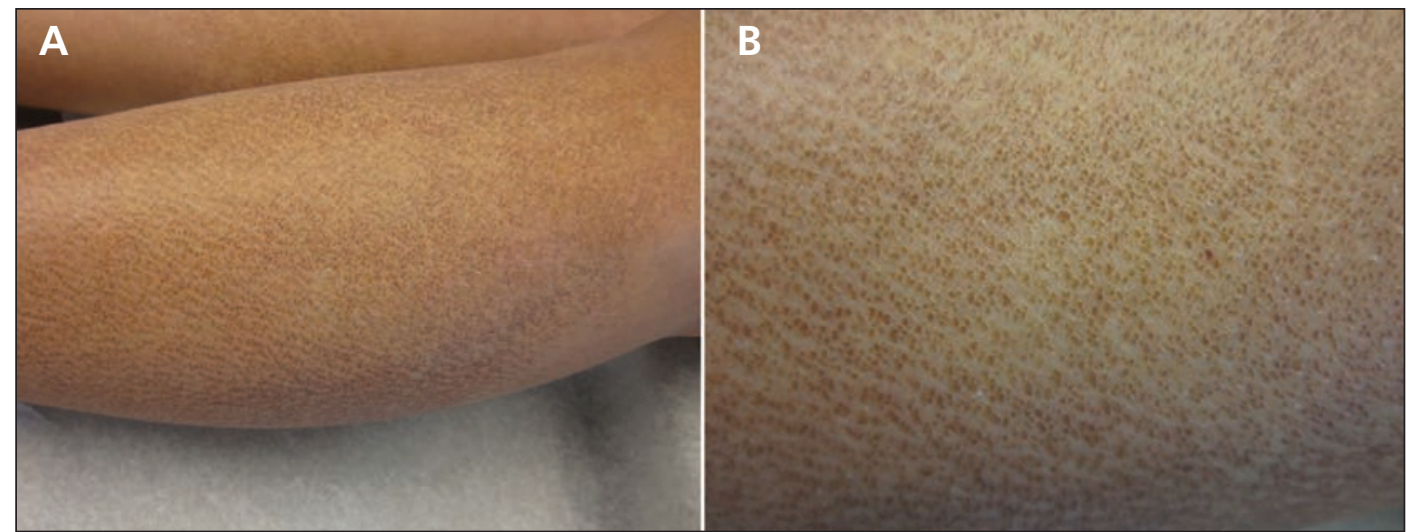

Figure 1: (A) Fine, pinpoint, tan-brown papules coalescing into a ripple pattern on the anterior surface of the lower shins of a 26-year-old woman. (B) A close-up view of the papules, diagnosed as lichen amyloidosis.

A 26-year-old woman presented to our clinic with an itchy rash on her legs that had persisted for 10 years. The rash had previously been treated with topical steroids and tazarotene cream, with no improvement. The patient's family history was noncontributory.

A physical examination showed discrete and coalescing hyperkeratotic tan-brown papules on the pretibial surfaces (Figure 1), consistent with lichen amyloidosis.

Lichen amyloidosis is the most common form of primary localized cutaneous amyloidosis. ${ }^{1}$ The cause is unknown, but the condition is thought to be induced by scratching. ${ }^{2}$ Owing to its benign nature, few studies have examined the condition's prevalence, but it typically presents in the 5th or 6th decade of life. ${ }^{3}$ The condition is more common in men and in people with darker skin phototypes. ${ }^{3}$ Although it is usually an isolated finding, lichen amyloidosis can be associated with multiple endocrine neoplasia type $2 \mathrm{~A} .^{4}$

Lichen amyloidosis typically presents as multiple pruritic, firm, hyperpigmented, hyperkeratotic papules on the shins that later coalesce to give the appearance of a rippled pattern.1,2 Over time, the papules can form thickened plaques that resist treatment. ${ }^{1,2}$ The differential diagnosis in cludes lichen simplex chronicus (i.e., chronically excoriated plaques with accentuation of skin lines), lichen sclerosis et atrophicus (i.e., pink inflammatory plaques with central white atrophic scarring) and prurigo nodularis (i.e., larger papules with hemorrhagic crusting). The diagnosis can usually be made clinically, particularly in patients with the classic presentation. A skin biopsy should be reserved for evolving lesions. ${ }^{1,2}$

Lichen amyloidosis is a chronic condition without potential for malignant transformation. Treatment is not required but can be employed for symptomatic or cosmetic complaints. ${ }^{2}$ Therapeutic options are limited, but success has been reported with topical or intralesional steroids, antihistamines, ultraviolet light therapy, laser therapy, dermabrasion, scalpel scraping and retinoid agents. ${ }^{2}$ Our patient underwent weekly narrowband ultraviolet $B$ phototherapy localized to her shins. Her pruritus resolved, but the lesions persisted.

\section{References}

1. Tay CH, Dacosta JL. Lichen Amyloidosus-clinical study of 40 cases. Br J Dermatol 1970;82:129-36.

2. Salim T, Shenoi SD, Balachandran C, et al. Lichen amyloidosus: a study of clinical, histopathologic and immunofluorescence findings in 30 cases. Indian J Dermatol Venereol Leprol 2005;71:166-9.

3. Chen JF, Chen YF. Dermacase. Can you identify this condition? Lichen amyloidosis. Can Fam Physician 2012;58:1233-5.

4. Verga U, Beck-Peccoz P, Cambiaghi S. Cutaneous lichen amyloidosis in multiple endocrine neoplasia type 2A. Thyroid 2002;12:1149. 